\title{
INDICABLE GROUPS AND ENDOMORPHIC PRESENTATIONS
}

\author{
MUSTAFA GÖKHAN BENLI \\ Department of Mathematics, Texas A\&M University, Mailstop 3368, College Station, TX 77843, USA \\ e-mail:mbenli@math.tamu.edu
}

(Received 12 July 2011; accepted 24 September 2011; first published online 12 December 2011)

\begin{abstract}
In this paper we look at presentations of subgroups of finitely presented groups with infinite cyclic quotients. We prove that if $H$ is a finitely generated normal subgroup of a finitely presented group $G$ with $G / H$ cyclic, then $H$ has ascending finite endomorphic presentation. It follows that any finitely presented indicable group without free semigroups has the structure of a semidirect product $H \rtimes \mathbb{Z}$, where $H$ has finite ascending endomorphic presentation.
\end{abstract}

2010 Mathematics Subject Classification. 20F05

1. Introduction. It is a well-known fact that finite index subgroups of finitely presented groups are also finitely presented. But once one looks at subgroups of infinite index, various possibilities can occur. It may be that the subgroup is not finitely generated but even one can have finitely generated infinitely presented subgroups. A well-known example is the kernel of the map $F_{2} \times F_{2} \rightarrow \mathbb{Z}$ where each generator is mapped to 1 (See [4]).

In this paper we look at subgroups of finitely presented groups with infinite cyclic quotients. The Higman embedding theorem [10] states that finitely generated subgroups of finitely presented groups are exactly the recursively presented groups. In the case where the subgroup has infinite cyclic quotient, we show that it has a special recursive presentation called a finite endomorphic presentation (or a finite L-presentation). More precisely we prove the following:

THEOREM 1. Let $G$ be a finitely presented group containing a finitely generated normal subgroup $H$ such that $G / H$ is infinite cyclic. Then, $H$ has ascending finite endomorphic presentation with two free group endomorphisms.

Intuitively, a finite endomorphic presentation is a generalisation of a finite presentation in which the relators of the presentation are obtained by iterating a finite set of initial relators over a finite set of endomorphisms of the underlying free group (see next section for a precise definition). It is yet another way of defining a group with finite data. Such presentations first arise in the study of self-similar groups: It was proven by Lysenok in [14] that the first Grigorchuk group $\mathcal{G}$ has the following presentation:

$$
\left.\mathcal{G}=\langle a, b, c, d| a^{2}, b^{2}, c^{2}, d^{2}, b c d, \sigma^{i}\left((a d)^{4}\right), \sigma^{i}\left((\text { adacac })^{4}\right), i \geq 0\right\rangle
$$


where $\sigma$ is the substitution,

$$
\sigma=\left\{\begin{array}{rlr}
a & \mapsto & a c a \\
b & \mapsto & d \\
c & \mapsto & b \\
d & \mapsto & c
\end{array} .\right.
$$

Later, more examples of presentations of this kind were found for various groups, including iterated monodromy groups (see for example $[\mathbf{1}, \mathbf{2}, \mathbf{7}, \mathbf{8}]$ ). A systematic study of such presentations was done by Bartholdi in [1], who also suggested the name endomorphic presentations. In the same paper it is also proved that any finitely generated, regular branch, self-similar group has such a presentation.

Groups with finite endomorphic presentations embed nicely in finitely presented groups obtained from the original group via finitely many HNN extensions [1]. The first example of such an embedding was done by Grigorchuk in [5] for group $\mathcal{G}$. Using Lysenok's presentation he showed that $\mathcal{G}$ embeds into the finitely presented HNN extension

$$
\overline{\mathcal{G}}=\left\langle\mathcal{G}, t \mid t^{-1} \mathcal{G} t=\sigma(\mathcal{G})\right\rangle
$$

which is amenable but not elementary amenable. This showed that amenable and elementary amenable groups are separated even in the class of finitely presented groups.

Recall that a group is termed indicable if it has a homomorphism onto the infinite cyclic group. Indicable groups play an important role in the study of right orderable groups, amenability and bounded cohomology (See $[\mathbf{1 1}, \mathbf{1 2}, \mathbf{1 5}])$.

Bieri and Strebel's theorem (p. 67 in [3]) states that a finitely presented indicable group not containing a free subgroup of rank 2, is an ascending HNN extension with a finitely generated base group. The group $\overline{\mathcal{G}}$ is amenable, hence cannot contain free subgroup on two generators. It is also indicable. Hence, it is a finitely presented indicable group, which is an ascending HNN extension with the finitely generated base group $\mathcal{G}$ that has finite endomorphic presentation. Motivated by this, Grigorchuk in [6] asked the following question:

Is it correct that a finitely presented indicable group not containing a free subgroup of rank 2 is an ascending HNN extension of a base group with finite endomorphic presentation?

As a corollary to Theorem 1, we provide an answer to this question under the stronger assumption that the group has no free semigroup of rank 2.

THEOREM 2. Let $G$ be a finitely presented indicable group not containing a free semigroup of rank 2. Then $G$ has the form of a semidirect product $H \rtimes \mathbb{Z}$, where $H$ has ascending finite endomorphic presentation.

The reason why we need the stronger assumption is that in this case the kernel of the homomorphism onto the infinite cyclic group itself is finitely generated and hence Theorem 1 can be applied.

\section{Definitions and preliminaries.}

\section{NotATION:}

- If $G$ is a group and $X$ a subset, then $\langle X\rangle$ denotes the subgroup of $G$ generated by $X$ and $\langle X\rangle^{\#}$ denotes the normal subgroup of $G$ generated by $X$. 
- $X^{ \pm}$stands for the set $X \cup X^{-}$.

- If $Y$ is a set of endomorphisms of a group, $Y^{*}$ stands for the free monoid generated by $Y$, i.e. the closure of $\{1\} \cup Y$ under composition.

- Unless stated otherwise, an equality means equality as words. We will indicate whenever necessary that some equality is thought to hold in some group.

- If $w$ is an element of the free group on a set $X$ and $x \in X, \exp _{x}(w)$ denotes the exponent sum of $x$ in $w$.

We will frequently use the following fact, also known as Dyck's theorem:

If $G$ is a group given as $F / N$, where $F$ is a free group and $N=\langle R\rangle^{\#}$ for some $R \subset F$, then any map

$$
\phi: F \longrightarrow H
$$

to another group $H$ satisfying $\phi(r)=1$ in $H$ for all $r \in R$ induces a well-defined group homomorphism

$$
\phi: G \longrightarrow H
$$

DEFINITION. An endomorphic presentation (or an L-presentation) is an expression

$$
\langle X|Q| R \mid \Phi\rangle
$$

where $X$ is a set, $Q, R$ are subsets of the free group $F(X)$ on the set $X$ and $\Phi$ is a set of endomorphisms of $F(X)$. Expression (1) defines a group

$$
G=F(X) / N
$$

where

$$
N=\left\langle Q \cup \bigcup_{\phi \in \Phi^{*}} \phi(R)\right\rangle^{\#}
$$

It is called a finite endomorphic presentation (or a finite L-presentation) if $X, Q, R, \Phi$ are all finite and ascending if $Q$ is empty. It is called invariant if the endomorphisms in $\Phi$ induce endomorphisms of $G$. Note that ascending Lpresentations are invariant, but not all finite L-presentations are invariant (see [9]).

(Some authors prefer to reserve the name L-presentation to the case where $\Phi$ only contains a single endomorphism. We will not make such a distinction and use both names.)

Clearly, all finite presentations are finite L-presentations. As mentioned in the Introduction there are groups (such as the Grigorchuk group), which are not finitely presented but finitely L-presented. Also, a counting argument shows that most groups are not finitely L-presented. For general properties of L-presentations see [1] and also the recent paper [9], where a variant of the Reidemeister-Schreier procedure is proven for finitely L-presented groups.

We cite some auxiliary lemmas, which we will use later. 
LEMMA 1. (see [13]). If a group $G$ has no free sub-semigroup of rank 2, then for all $a, b \in G$ the subgroup

$$
\left\langle b^{-n} a b^{n} \mid n \in \mathbb{Z}\right\rangle
$$

is finitely generated.

LEMMA 2. (see [16]). Let $G$ be a finitely generated group and $H$ a normal subgroup such that $G / H$ is solvable. If for all $a, b \in G$ the subgroup $\left\langle b^{-n} a b^{n} \mid n \in \mathbb{Z}\right\rangle$ is finitely generated, then $H$ is finitely generated.

Lemmas 1 and 2 together give the following.

LEMMA 3. Let $G$ be a finitely generated group not containing free sub-semigroup of rank 2. If $G / H$ is solvable, then $H$ is finitely generated.

\section{Proof of theorems.}

THEOREM 1. Let $G$ be a finitely presented group. Let $H$ be a finitely generated normal subgroup such that $G / H$ is infinite cyclic. Then $H$ has ascending finite L-presentation with two free group endomorphisms.

Proof. Suppose that for $t \in G$ we have $G / H=\langle t H\rangle$, then $G$ has the form of a semidirect product $G=H \rtimes\langle t\rangle$.

It follows from Neumann's Theorem (p. 52 in [3]) that $G$ has a presentation of the form

$$
G=\left\langle t, a_{1}, \ldots, a_{m} \mid r_{1}, \ldots, r_{n}\right\rangle
$$

where

$$
H=\left\langle a_{1}, \ldots, a_{m}\right\rangle_{G}^{\#}
$$

and

$$
\exp _{t}\left(r_{k}\right)=0
$$

Consequently, the set

$$
T=\left\{t^{i} \mid i \in \mathbb{Z}\right\}
$$

is the right Schreier transversal for $H$ in $G$.

Following the Reidemeister-Schreier process for $H$, we can take the elements

$$
a_{j, i}=t^{-i} a_{j} t^{i} \quad j=1, \ldots, m i \in \mathbb{Z}
$$

as generators for $H$ and the words

$$
r_{k, i}=\rho\left(t^{-i} r_{k} t^{i}\right) \quad k=1, \ldots, n i \in \mathbb{Z}
$$

as relators, where $\rho$ is the rewriting of $t^{-i} r_{k} t^{i}$ as a word in $a_{j, i}$ s. So $H$ has the presentation,

$$
H=\left\langle a_{j, i} \quad(j=1, \ldots, m \quad i \in \mathbb{Z}) \mid r_{k, i} \quad(k=1, \ldots, n \quad i \in \mathbb{Z})\right\rangle .
$$


Each $r_{k}$ is a word of the form

$$
r_{k}=\prod_{s=1}^{n_{k}} t^{-l_{s}} a_{z_{s}} t^{l_{s}}
$$

where $a_{z_{s}} \in\left\{a_{j}, j=1, \ldots, m\right\}^{ \pm}$and $n_{k} \in \mathbb{N}, l_{s} \in \mathbb{Z}$. Therefore, we have

$$
r_{k, 0}=\rho\left(r_{k}\right)=\rho\left(\prod_{s=1}^{n_{k}} t^{-l_{s}} a_{z_{s}} t^{l_{s}}\right)=\prod_{s=1}^{n_{k}} a_{z_{s}, l_{s}}
$$

and

$$
r_{k, i}=\rho\left(t^{-i} r_{k} t^{i}\right)=\prod_{s=1}^{n_{k}} a_{z_{s}, l_{s}+i} \quad i \in \mathbb{Z} .
$$

The map

$$
s: H \longrightarrow H
$$

defined by $s(h)=t^{-1} h t$ is clearly an automorphism of $H$. With respect to presentation (2) of $H, s$ becomes $s\left(a_{j, i}\right)=a_{j, i+1}$.

Let $F$ be the free group on $\left\{a_{j, i} \quad j=1, \ldots, m i \in \mathbb{Z}\right\}$. We will again denote by $s$ the automorphism of $F$ sending $a_{j, i}$ to $a_{j, i+1}$.

Since by assumption $H$ is finitely generated, we can select a big enough natural number $N$ with the following properties:

- $H=\left\langle a_{j, i} \quad(j=1, \ldots, m) \quad|i| \leq N\right\rangle$.

- Each word $r_{k, 0}$ is a word in $\left\{a_{j, i} \quad j=1, \ldots, m \quad|i| \leq N\right\}^{ \pm}$.

So, each $a_{j, i}$ can be represented by a word in the finite generating set $\left\{a_{j, i} \quad j=\right.$ $1, \ldots, m \quad|i| \leq N\}^{ \pm}$.

For each $a_{j, i}$ we will recursively construct a word $\gamma\left(a_{j, i}\right)$ in this new finite generating set, which represents $a_{j, i}$ in $H$.

For $a_{j, i}$ with $|i| \leq N$ we simply define $\gamma\left(a_{j, i}\right)$ to be $a_{j, i}$.

Pick $\gamma\left(a_{j, N+1}\right)$ and $\gamma\left(a_{j,-(N+1)}\right)$ two words in $\left\{a_{j, i}|j=1, \ldots, m \quad| i \mid \leq N\right\}^{ \pm}$ representing $a_{j, N+1}$ and $a_{j,-(N+1)}$ in $H$, respectively.

For $i \geq N+1$ we define $\gamma\left(a_{j, i+1}\right)$ recursively as follows:

$$
\gamma\left(a_{j, i+1}\right)=\gamma\left(s\left(\gamma\left(a_{j, i}\right)\right)\right)
$$

(for a word $w$, we define $\gamma(w)$ as the word obtained by applying $\gamma$ to each letter of $w$ ). Note that $s\left(\gamma\left(a_{j, i}\right)\right)$ is a word in $\left\{a_{j, i}|j=1, \ldots, m \quad| i \mid \leq N+1\right\}^{ \pm}$, therefore we can apply $\gamma$ to it.

Similarly, for $i \leq-(N+1)$ we define $\gamma\left(a_{j, i-1}\right)$ as

$$
\gamma\left(a_{j, i-1}\right)=\gamma\left(s^{-1}\left(\gamma\left(a_{j, i}\right)\right)\right) .
$$

Defining $\gamma$ as above gives the following equalities in the free group $F$ :

$$
\gamma\left(a_{j, i+1}\right)=\gamma\left(s\left(\gamma\left(a_{j, i}\right)\right)\right) \text { for } i \geq-N
$$


and

$$
\gamma\left(a_{j, i-1}\right)=\gamma\left(s^{-1}\left(\gamma\left(a_{j, i}\right)\right)\right) \text { for } \quad i \leq N
$$

\section{LEMMA 4. H has the presentation}

$$
\left\langle a_{j, i}(j=1, \ldots, m \quad|i| \leq N) \mid \gamma\left(r_{k, i}\right)(k=1, \ldots, n \quad i \in \mathbb{Z})\right\rangle .
$$

Proof. This follows by the Tietze transformations, but we will explicitly construct an isomorphism between these presentations. In order to avoid confusion, we denote elements in the asserted presentation with bars and set

$$
\bar{H}=\left\langle\overline{a_{j, i}}(j=1, \ldots, m \quad|i| \leq N) \mid \overline{\gamma\left(r_{k, i}\right)}(k=1, \ldots, n \quad i \in \mathbb{Z})\right\rangle .
$$

We will show that $\bar{H} \cong H$ using the presentation (2) of $H$. For this define:

$$
\begin{aligned}
\varphi: H & \left.\longrightarrow \frac{\bar{H}}{a_{j, i}}\right) \frac{}{\gamma\left(a_{j, i}\right)} .
\end{aligned}
$$

We have $\varphi\left(r_{k, i}\right)=\overline{\gamma\left(r_{k, i}\right)}=1$ in $\bar{H}$. So $\varphi$ maps relators of $H$ to relators in $\bar{H}$ and hence is a well-defined group homomorphism. Conversely, define

$$
\psi: \overline{\bar{H}} \longrightarrow H
$$

Since $\gamma\left(a_{j, i}\right)=a_{j, i}$ in $H$, we have

$$
\psi\left(\overline{\gamma\left(r_{k, i}\right)}\right)=\gamma\left(r_{k, i}\right)=r_{k, i}=1 \quad \text { in } H,
$$

which shows that $\psi$ is a well-defined group homomorphism.

Finally, the following equalities show that $\varphi$ and $\psi$ are mutual inverses:

$$
(\varphi \circ \psi)\left(\overline{a_{j, i}}\right)=\varphi\left(a_{j, i}\right)=\overline{\gamma\left(a_{j, i}\right)}=\overline{a_{j, i}}
$$

(where the last equality is true, since $|i| \leq N$ in this case),

$$
(\psi \circ \varphi)\left(a_{j, i}\right)=\psi\left(\overline{\gamma\left(a_{j, i}\right)}\right)=\gamma\left(a_{j, i}\right)=a_{j, i} \quad \text { in } \quad H .
$$

Hence, $\bar{H}$ is isomorphic to $H$.

Let $F_{r}$ be the free group with generators $\left\{a_{j, i}|j=1, \ldots, m \quad| i \mid \leq N\right\}$. Define two endomorphisms $\eta$ and $\tau$ of $F_{r}$ as follows:

$$
\eta\left(a_{j, i}\right)=\gamma\left(s\left(a_{j, i}\right)\right)=\gamma\left(a_{j, i+1}\right)
$$

and

$$
\tau\left(a_{j, i}\right)=\gamma\left(s^{-1}\left(a_{j, i}\right)\right)=\gamma\left(a_{j, i-1}\right),
$$

where $\gamma$ is as above. Note that $\eta$ and $\tau$ induce the automorphisms $s$ and $s^{-1}$ of $H$, respectively. 
LEMMA 5. In $F_{r}$ we have the equality

$$
\gamma\left(r_{k, i}\right)=\left\{\begin{array}{ll}
\eta^{i}\left(r_{k, 0}\right) & \text { if } \quad i \geq 0 \\
\tau^{-i}\left(r_{k, 0}\right) & \text { if } \quad i<0
\end{array} .\right.
$$

Proof. Suppose $i \geq 0$. We use induction on $i$.

If $i=0, \gamma\left(r_{k, 0}\right)=r_{k, 0}$ by choice of $\gamma$ and the natural number $N$. Suppose the equality holds for $i$. Then,

$$
\begin{aligned}
\eta^{i+1}\left(r_{k, 0}\right) & =\eta\left(\eta^{i}\left(r_{k, 0}\right)\right) \\
& =\eta\left(\gamma\left(r_{k, i}\right)\right) \quad \text { (by induction hypothesis) } \\
& =\eta\left(\gamma\left(\prod a_{z_{s}, l_{s}+i}\right)\right) \quad \text { (using (3)) } \\
& =\prod \eta\left(\gamma\left(a_{z_{s}, l_{s}+i}\right)\right) \\
& =\prod \gamma s \gamma\left(a_{z_{s}, l_{s}+i}\right) \\
& =\prod \gamma\left(a_{z_{s}, l_{s}+i+1}\right) \quad\left(\text { using }(4), \text { since }\left|l_{s}\right| \leq N\right) \\
& =\gamma\left(\prod a_{z_{s}, l_{s}+i+1}\right) \\
& =\gamma\left(r_{k, i+1}\right) .
\end{aligned}
$$

A similar argument with induction on $-i$ (and using (5)) shows the required identity for $i<0$.

LEMMA 6. $H$ has the following ascending finite L-presentation:

$$
\left\langle a_{j, i} \quad(j=1, \ldots, m \quad|i| \leq N)\left|r_{k, 0} \quad k=1, \ldots, n\right|\{\eta, \tau\}\right\rangle .
$$

Proof. Again, not to cause confusion we denote the asserted presentation with bars and set

$$
\bar{H}=\left\langle\overline{a_{j, i}} \quad(j=1, \ldots, m \quad|i| \leq N)\left|\overline{r_{k, 0}} \quad k=1, \ldots, n\right|\{\bar{\eta}, \bar{\tau}\}\right\rangle,
$$

where $\bar{\eta}, \bar{\tau}$ are endomorphisms of the free group $\overline{F_{r}}$ analogous to $\eta$ and $\tau$. More precisely,

$$
\begin{aligned}
& \bar{\eta}\left(\overline{a_{j, i}}\right)=\overline{\eta\left(a_{j, i}\right)}, \\
& \bar{\tau}\left(\overline{a_{j, i}}\right)=\overline{\tau\left(a_{j, i}\right)} .
\end{aligned}
$$

We will show that $\bar{H} \cong H$ and we will use the presentation of $H$

$$
\left\langle a_{j, i}(j=1, \ldots, m \quad|i| \leq N) \mid \gamma\left(r_{k, i}\right)(k=1, \ldots, n \quad i \in \mathbb{Z})\right\rangle,
$$

which was found in Lemma 4. To this end define:

$$
\begin{aligned}
\phi: H & \longrightarrow \bar{H} \\
a_{j, i} & \mapsto \overline{a_{j, i}} .
\end{aligned}
$$


We have

$$
\phi\left(\gamma\left(r_{k, i}\right)\right)=\overline{\gamma\left(r_{k, i}\right)}= \begin{cases}\bar{\eta}^{i}\left(\overline{r_{k, 0}}\right) & \text { if } \quad i \geq 0 \\ \bar{\tau}^{-i}\left(\overline{r_{k, 0}}\right) & \text { if } \quad i<0\end{cases}
$$

by lemma 5 . Hence, $\phi$ is a well-defined group homomorphism. Conversely, define:

$$
\chi: \bar{H} \longrightarrow \begin{gathered}
H \\
\overline{a_{j, i}} \mapsto a_{j, i}
\end{gathered}
$$

To show that $\chi$ is well defined, we need to prove that for all $\bar{f} \in\{\bar{\eta}, \bar{\tau}\}^{*}$ and for all $k=1, \ldots, n$ we have

$$
\chi\left(\bar{f}\left(\overline{r_{k, 0}}\right)\right)=1 \text { in } H .
$$

This is true since $\eta$ and $\tau$ (and hence $f$ ) induce isomorphisms on $H$. This shows that $\chi$ is a well-defined group homomorphism. Clearly, $\phi$ and $\chi$ are mutual inverses.

Hence, we have proven Theorem 1.

THEOREM 2. Let $G$ be a finitely presented indicable group not containing a free semigroup of rank 2. Then, $G$ has the form of a semi-direct product $H \rtimes \mathbb{Z}$, where $H$ has ascending finite L-presentation.

Proof. Follows directly from Theorem 1 and Lemma 3.

\section{Some Remarks.}

(1) As mentioned in the Introduction, groups with invariant finite L-presentations embed nicely into finitely presented groups via HNN extensions. In our special case the endomorphisms of the L-presentation of $H$ actually induce automorphism of $H$ and $H$ embeds into $G$ as a normal subgroup.

(2) Though all finitely generated recursively presented groups embed into finitely presented groups, I have been told by Mark Sapir (private communication) that not all finitely generated, recursively presented groups embed into finitely presented groups as normal subgroups. His example was the first Grigorchuk group. This shows that even finitely L-presented groups may fail to be normal subgroups of finitely presented groups. This indicates that such groups have a rather restricted structure. Hence, a natural question is, what additional structure finitely generated normal subgroups of finitely presented groups have? One answer could be given if one can generalise Theorem 1 to arbitrary finitely generated normal subgroups. One would obtain characterisation in the following sense:

A finitely generated group is a normal subgroup of a finitely presented group if and only if it has an ascending finite L-presentation where the endomorphisms induce automorphisms of the group.

Therefore, we would like to formulate the question whether Theorem 1 can be generalised to arbitrary finitely generated normal subgroups.

(3) We would like to present a concrete example in which Theorem 1 can be used. This is also a counter example to the assertion (as written in Theorem 2.16 in [1]) that all finitely L-presented groups have the Schur Multiplier, the direct product of finitely 
generated abelian groups. Upon discussing with Bartholdi [1], it was observed that one needs one additional hypothesis.

Let $G$ be the group given by the presentation

$$
\left\langle a, b, t, u \mid[a, b],[a, u],[t, b],[t, u], a^{t}=a^{2}, b^{u}=b^{2}\right\rangle,
$$

which is the direct square of the Baumslag-Solitar group $B S(1,2)$. Let $z=t u^{-1}$ and consider the subgroup $H=\langle a, b, z\rangle$, which is normal and has infinite cyclic quotient. Then, following Theorem 1 one arrives at the following finite L-presentation for $H$ :

$$
\left\langle a, b, z\left|[a, b], a^{z}=a^{2},\left(b^{2}\right)^{z}=b\right|\{\eta, \tau\}\right\rangle,
$$

where

$$
\eta=\left\{\begin{array}{lll}
a & \mapsto & a^{2} \\
b & \mapsto & b \\
z & \mapsto & z
\end{array}\right.
$$

and

$$
\tau=\left\{\begin{array}{ccc}
a & \mapsto & z a z^{-1} \\
b & \mapsto & b \\
z & \mapsto & z
\end{array}\right.
$$

Now, since $B S(1,2)=\mathbb{Z}\left[\frac{1}{2}\right] \rtimes \mathbb{Z}$, we have $H=\mathbb{Z}\left[\frac{1}{2}\right]^{2} \rtimes \mathbb{Z}$ and using Shapiro's lemma one can see that $H_{2}(H, \mathbb{Z}) \cong \mathbb{Z}\left[\frac{1}{2}\right]$.

(4) Another problem of interest is the structure of subgroups of finitely L-presented groups. For finite index subgroups one has the Reidemeister-Schreier algorithm to compute a finite L-presentation for the subgroup (see [9]). For other subgroups it would be nice to investigate whether analogous statements similar to Theorem 1 hold.

ACKNOWLedGements. I wish like to thank my advisor Rostislav Grigorchuk for his valuable comments and helpful discussions. I also want to thank Laurent Bartholdi for nice remarks and suggestions. I am grateful to Ben Wieland for suggesting example in Remark 3.

\section{REFERENCES}

1. L. Bartholdi, Endomorphic presentations of branch groups, J. Algebra, 268(2) (2003), 419-443. ISSN 0021-8693.

2. L. Bartholdi, R. I. Grigorchuk and Z. Šuniḱ, Branch groups, in Handbook of algebra, vol. 3 (Hazewinkel M., Editor) (North-Holland, Amsterdam, Netherlands, 2003), 989-1112.

3. G. Baumslag, Topics in combinatorial group theory, Lectures in Mathematics ETH Zürich (Birkhäuser Verlag, Basel, Switzerland, 1993). ISBN 3-7643-2921-1.

4. G. Baumslag and J. E. Roseblade, Subgroups of direct products of free groups, J. London Math. Soc. (2) 30(1) (1984), 44-52. ISSN 0024-6107.

5. R. I. Grigorchuk, An example of a finitely presented amenable group that does not belong to the class EG, Mat. Sb. 189(1) (1998), 79-100. ISSN 0368-8666.

6. R. Grigorchuk, Solved and unsolved problems around one group, in Infinite groups: Geometric, combinatorial and dynamical aspects, vol. 248 of Progress in Mathematics (Bartholdi L., Checcherini-Silberstein T., Smirnova-Nagnibeda T. and Zuk A., Editors) (Birkhäuser, Basel, 2005), 117-218. 
7. R. Grigorchuk, D. Savchuk and Z. Šunić, The spectral problem, substitutions and iterated monodromy in Probability and mathematical physics, vol. 42 of CRM proceedings \& lecture notes (Dawson D. A., Jaksic V. and Vainberg B., Editors) (American Mathematical Society, Providence, RI, 2007), 225-248.

8. R. I. Grigorchuk and A. Żuk, Spectral properties of a torsion-free weakly branch group defined by a three state automaton, in Computational and statistical group theory, vol. 298 of Contemporary mathematics series (American Mathematical Society, Providence, RI, 2002), $57-82$.

9. R. Hartung, A Reidemeister-Schreier theorem for finitely 1-presented groups. URL: http://arxiv.org/abs/1108.2403. 455-475.

10. G. Higman, Subgroups of finitely presented groups, Proc. Roy. Soc. Ser. A 262 (1961),

11. J. Howie, On locally indicable groups, Math. Z. 180(4) (1982), 445-461. ISSN 0025-5874.

12. P. H. Kropholler, Amenability and right orderable groups, Bull. Lond. Math. Soc. 25(4) (1993), 347-352. ISSN 0024-6093.

13. P. Longobardi, M. Maj and A. H. Rhemtulla, Groups with no free subsemigroups, Trans. Am. Math. Soc. 347(4) (1995), 1419-1427. ISSN 0002-9947.

14. I. G. Lysënok, A set of defining relations for the Grigorchuk group, Mat. Zametki. 38 (4) (1985), 503-516, 634. ISSN 0025-567X.

15. D. W. Morris, Amenable groups that act on the line, Algebr. Geom. Topol. 6 (2006), 2509-2518. ISSN 1472-2747.

16. S. Rosset, A property of groups of non-exponential growth, Proc. Am. Math. Soc. 54 (1976), 24-26. ISSN 0002-9939. 